\title{
Causality of Phase of Wave Function or Can Copenhagen Interpretation of Quantum Mechanics Be Considered Complete?
}

\author{
Ivan Georgiev Koprinkov \\ Department of Applied Physics, Technical University of Sofia, Sofia, Bulgaria \\ Email: igk@tu-sofia.bg
}

Received 11 December 2015; accepted 26 February 2016; published 29 February 2016

Copyright @ 2016 by author and Scientific Research Publishing Inc.

This work is licensed under the Creative Commons Attribution International License (CC BY). http://creativecommons.org/licenses/by/4.0/

c) (i) Open Access

\begin{abstract}
Theoretical and experimental evidences of a causal relation of the phase of the wave function and physical reality are presented. The Copenhagen interpretation of quantum mechanics, which gives physical meaning to the amplitude of the wave function only, cannot be considered complete on that ground. A new dynamics-statistical interpretation of quantum mechanics is proposed.
\end{abstract}

\section{Keywords}

Quantum Mechanics, Wave Function, Material Phase, Causality, Interpretation

\section{Introduction}

The quantum mechanics appears to be the most successful theory of physical reality at a microscopic level. Nevertheless, it is a subject of intensive debates mainly in the field of interpretation. The physical state in quantum mechanics is described not by a definite number of dynamical variables, coordinates, linear momenta, etc., as in the classical mechanics, but by an abstract quantity - the wave function. Although it describes the state of localized physical objects, i.e., particle or system of particles, the wave function (quantum state) is distributed in space as for the continuous objects, i.e., waves. Thus, particle-wave duality or locality versus non-locality is built in the foundations of quantum mechanics. The description of the state of a localized physical object by a substantially non-local quantity - the wave function, does not have an analog in the classical physics. That is why, the physical meaning of the wave function is not obvious and its relation to physical reality is a fundamental problem.

Since Einstein's belief that "God does not play dice” was not adopted, the Copenhagen probabilistic interpretation has been widely accepted. It, however, still remains unsatisfactory for part of the physicists and a number of alternative interpretations have been proposed. The Copenhagen interpretation attributes probabilistic physical 
meaning to the amplitude of the wave function only, while its (dynamical) phase, hereafter referred to as material phase (MP), is considered, in principle, as unobservable. Occasionally, the phase difference is considered concerning mainly the interference phenomena but not the interpretation of quantum mechanics. Thus, the role of the MP is neglected or, at least, strongly underestimated in the standard quantum mechanics. Einstein, Podolsky, and Rosen (EPR) attempted to show in a gedanken experiment that the quantum mechanics is an incomplete theory and the quantum phenomena can be completely specified in terms of hidden variables [1]. J. S. Bell has shown [2] that the predictions of the quantum mechanics and the hidden variable theories based on Einstein's understanding of locality and realism can be distinguished experimentally. The real EPR type experiments reveal the non-locality of the quantum phenomena although it seems that the local realism cannot be ruled out yet decisively due to some experimental "loopholes" [3]. It, however, does not exclude the opposite type of "incompleteness", i.e., the wave function may contain substantially more information than it is formally recognized by the quantum mechanics in its Copenhagen interpretation. In this work we show that the relation of the wave function with the physical reality is not restricted to the amplitude only but the MP becomes causally involved in the physical phenomena [4]. A new dynamics-statistical interpretation of quantum mechanics is introduced on that ground.

\section{Evidences of Material Phase Causality}

The basic arguments in support of MP causality will be classified as: special theoretical arguments, general theoretical arguments, and experimental evidences.

\subsection{Special Theoretical Arguments}

The problem of MP causality is treated here within an analytic solution of the time dependent Schrödinger equation. To reveal the MP dynamics, the quantum system is involved in a definite physical process, in our case, interaction with electromagnetic field and environment (damping), described by the Hamiltonian

$\hat{H}=\sum_{j=1}^{2} \hbar \omega_{j}|j\rangle\langle j|-\mu E(|1\rangle\langle 2|+$ h.c. $)-i \hbar \sum_{j=1}^{2}\left(\gamma_{j} / 2\right)|j\rangle\langle j|$ [5]. New quantum states, named phase-sensitive nonadiabatic dressed states (PSNADSs) [4], are derived in that case including the contribution of phase and nonadiabatic factors from the field and the environment. Ground $|G\rangle=\operatorname{COS}(\theta / 2)\left|G_{R}\right\rangle+\operatorname{SIN}(\theta / 2)\left|G_{V}\right\rangle$ and excited $|E\rangle=\operatorname{COS}(\theta / 2)\left|E_{R}\right\rangle-\operatorname{SIN}(\theta / 2)\left|E_{V}\right\rangle$ PSNADSs consist of real, $\left|G_{R}\right\rangle,\left|E_{R}\right\rangle$, and virtual, $\left|G_{V}\right\rangle,\left|E_{V}\right\rangle$, components: $\left|G_{R}\right\rangle=|g\rangle \exp \left(-i \Phi_{G R}\right),\left|G_{V}\right\rangle=|e\rangle \exp \left(-i \Phi_{G V}\right),\left|E_{R}\right\rangle=|e\rangle \exp \left(-i \Phi_{E R}\right),\left|E_{V}\right\rangle=|g\rangle \exp \left(-i \Phi_{E V}\right)$, where $\Phi_{G R}, \Phi_{G V}, \Phi_{E R}, \Phi_{E V}$ are the total MPs of the respective states, and $|g\rangle$, $|e\rangle$ are the bare states of the quantum system from which PDNADSs originate. The MPs of the PSNADSs at ground state, Equations (1) left column, and at excited state, Equations (1) right column, initial conditions are [4]:

$$
\begin{array}{ll}
\Phi_{G R}=\varphi_{g}+\int_{0}^{t} \tilde{\omega}_{G}^{\prime} \mathrm{d} t^{\prime} & \Phi_{E R}=\varphi_{e}+\varphi(t)-\varphi_{0}+\int_{0}^{t} \tilde{\omega}_{E}^{\prime} \mathrm{d} t^{\prime} \\
\Phi_{G V}=\Phi_{G R}+\Phi_{F}=\varphi_{g}+\varphi(t)+\int_{0}^{t}\left(\tilde{\omega}_{G}^{\prime}+\omega\right) \mathrm{d} t^{\prime} & \Phi_{E V}=\Phi_{E R}-\Phi_{F}=\varphi_{e}-\varphi_{0}+\int_{0}^{t}\left(\tilde{\omega}_{E}^{\prime}-\omega\right) \mathrm{d} t^{\prime} \\
\Phi_{E R}=\Phi_{G V}+\Phi_{N A D}=\varphi_{g}+\varphi(t)+\int_{0}^{t} \tilde{\omega}_{E}^{\prime} \mathrm{d} t^{\prime} & \Phi_{G R}=\Phi_{E V}-\Phi_{N A D}=\varphi_{e}-\varphi_{0}+\int_{0}^{t} \tilde{\omega}_{G}^{\prime} \mathrm{d} t^{\prime} \\
\Phi_{E V}=\Phi_{E R}-\Phi_{F}=\varphi_{g}+\int_{0}^{t}\left(\tilde{\omega}_{E}^{\prime}-\omega\right) \mathrm{d} t^{\prime} & \Phi_{G V}=\Phi_{G R}+\Phi_{F}=\varphi_{e}+\varphi(t)-\varphi_{0}+\int_{0}^{t}\left(\tilde{\omega}_{G}^{\prime}+\omega\right) \mathrm{d} t^{\prime}
\end{array}
$$

where $\tilde{\omega}_{G}^{\prime}$, $\tilde{\omega}_{E}^{\prime}$ and $\omega$ are the frequencies of ground, excited PSNADSs and field, respectively, $\Delta \tilde{\omega}_{N A D}^{\prime}=$ $\tilde{\omega}_{E}^{\prime}-\tilde{\omega}_{G}^{\prime}-\omega$ is nonadiabatic frequency detuning, $\Phi_{N A D}=\int_{0}^{t} \Delta \tilde{\omega}_{N A D}^{\prime} \mathrm{d} t^{\prime}$ is nonadiabatic phase, $\varphi_{g}, \varphi_{e}$ are initial phases of the bare states, $\Phi_{F}(t)=\omega t+\varphi(t)$ and $\varphi(t)$ are total and carrier-envelope phases of the field, and $\varphi_{0}=\varphi(t=0)$.

The MP dynamics can be understood from a causal point of view based on the expressions for MP, Equations (1). Starting from $\left|G_{R}\right\rangle$, ground state initial condition or $|g\rangle--\rightarrow\left|G_{R}\right\rangle \rightarrow\left|G_{V}\right\rangle \Rightarrow\left|E_{R}\right\rangle \rightarrow\left|E_{V}\right\rangle$ sequence of creation of the PDNADSs from the bare ground state $|g\rangle$, Equations (1), Figure 1(a), the following evolution of the states and their phases along with the relevant physical processes can be found. The bare ground state 


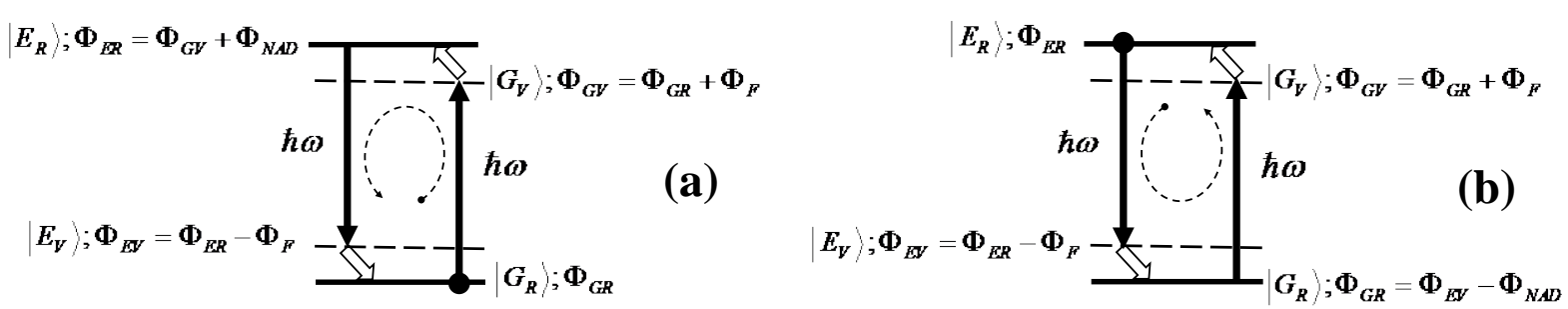

Figure 1. Material phase evolution within PSNADSs at ground (a) and excited (b) state initial conditions.

$|g\rangle$, forced by the electromagnetic field, evolves toward a new ground state-the PSNADS $|G\rangle$. It modifies the eigenfrequency $\omega_{g}$ of $|g\rangle$ to form the eigenfrequency $\tilde{\omega}_{G}^{\prime}$ of the real component $\left|G_{R}\right\rangle$ of ground state $|G\rangle$. The new ground state of eigenfrequency $\tilde{\omega}_{G}^{\prime}$ acquires phase $\int_{0}^{t} \tilde{\omega}_{G}^{\prime} \mathrm{d} t^{\prime}$ that is added to the initial phase $\varphi_{g}$, thus forming the total phase $\Phi_{G R}$ of $\left|G_{R}\right\rangle$, see Equations (1). Once $\left|G_{R}\right\rangle$ is created, the process of creation/population of other PSNADS components and their phases can be traced out starting from $\left|G_{R}\right\rangle$. The phase $\Phi_{G V}$ of the virtual component $\left|G_{V}\right\rangle$ results from the phase $\Phi_{G R}$ of the real component $\left|G_{R}\right\rangle$ adding the field phase $\Phi_{F}$. At the same time, physically, the virtual component of the ground state results from the real component of the ground state by a temporal association and reemission of one photon from the electromagnetic field. In the formation of $\left|E_{R}\right\rangle$ from $\left|G_{V}\right\rangle$, the phase $\Phi_{E R}$ of the real component $\left|E_{R}\right\rangle$ results from the phase $\Phi_{G V}$ of the virtual component $\left|G_{V}\right\rangle$ adding the nonadiabatic phase $\Phi_{N A D}$ acquired while the quantum system "overcomes" the frequency detuning $\Delta \tilde{\omega}_{N A D}^{\prime}$. According to the adiabatic theorem, the physical transition between different states, in this case $\left|G_{V}\right\rangle$ and $\left|E_{R}\right\rangle$, results from the nonadiabatic factors acting on the quantum system. It is accompanied by irreversible absorption of one photon from the field leading to population of the real excited state $\left|E_{R}\right\rangle$. Finally, the phase $\Phi_{E V}$ of the virtual component $\left|E_{V}\right\rangle$ results from the phase $\Phi_{E R}$ of the real component $\left|E_{R}\right\rangle$ subtracting the field phase $\Phi_{F}$. Physically, the virtual component of the excited state results from the real component of the excited state by a temporal emission and reabsorption of one photon from the quantum system. Similar behavior takes place at excited state initial condition or $|e\rangle--\rightarrow\left|E_{R}\right\rangle \rightarrow\left|E_{V}\right\rangle \Rightarrow\left|G_{R}\right\rangle \rightarrow\left|G_{V}\right\rangle$ sequence of creation of the PDNADSs starting from bare excited state $|e\rangle$, Equations (1), Figure 1(b). Thus, the dynamics of the quantum system within the PSNADSs reveals exact correspondence between the MP behavior and the accompanying physical processes. The MP behaves as an additive dynamical quantity that causally follows the initial conditions and the physical process responsible for the formation/population of given quantum state.

\subsection{General Theoretical Arguments}

The causal relation of the MP with the physical reality can be proved by general theoretical arguments. In polar representation, the wave function $\Psi=R(\boldsymbol{r}, t) \exp [-i \Phi(\boldsymbol{r}, t)]$ consists of amplitude $R(\boldsymbol{r}, t)$ and phase $\Phi(\boldsymbol{r}, t)=-\hbar^{-1} S(\boldsymbol{r}, t)$, where $S(\boldsymbol{r}, t)$ is the physical action. In other words, up to a constant factor, the MP $\Phi$ coincides with the physical action $S$. Substitution of the wave function $\Psi=R(\boldsymbol{r}, t) \exp [-i \Phi(\boldsymbol{r}, t)]$ in the Schrödinger equation leads to coupled differential equations for the action/phase and the amplitude

$$
\begin{gathered}
\partial S / \partial t+(\nabla S)^{2} / 2 m+V(\boldsymbol{r}, t)-\left(\hbar^{2} / 2 m\right)(\Delta R / R)=0 \\
\partial(R)^{2} / \partial t+\nabla \cdot\left(R^{2} \nabla S / m\right)=0
\end{gathered}
$$

Equations (2) and (3) constitute hydrodynamic representation of quantum mechanics. It is exploited by D. Bohm [6] in his ontological approach based on hidden variables concept [1]. Equation (2) is quantum mechanical equation of Hamilton-Jacobi for the action/phase. Equation (3) is continuity equation for quantum probability density $\rho=R^{2}=|\Psi|^{2}$. In Bohmian mechanics, the hydrodynamic representation is used to build a deterministic quantum theory based on the understanding of reality of the wave function. In our work, the hydrodynamic representation is used to reveal a fundamental relation between the phase and the amplitude of the wave function and to prove on that ground the MP causality. Really, the phase/action $\Phi / S$ and the amplitude $R$ obey coupled differential Equations (2), (3). Therefore, they are not independent but codetermine each other. Consequently, $S / \Phi \leftrightarrow R$ relation of one and the same wave function exists. Such relation is of general type that 
cannot be expressed in a closed form, excepting perhaps for some simple cases. Taking into account $S / \Phi \leftrightarrow R$ relation and the widely recognized $R \leftrightarrow$ physical reality relation (Copenhagen interpretation, Bohmian mechanics, etc.), existence of a complete relation can be established

$$
S(\boldsymbol{r}, t) / \Phi(\boldsymbol{r}, t) \leftrightarrow R(\boldsymbol{r}, t) \leftrightarrow \text { physical reality } .
$$

Such $S(\boldsymbol{r}, t) / \Phi(\boldsymbol{r}, t) \leftrightarrow R(\boldsymbol{r}, t) \leftrightarrow$ physical reality relation is founded in the quantum mechanics but it does not appear as a consequence of interpretation. It, however, can be used as a basic reference point in the interpretation of quantum mechanics. The relation (4) is the most important, to our opinion, theoretical argument of MP causality. It is completely general because it rests solely on the structure of Schrödinger equation and wave function. The hydrodynamic representation is well known since the early days of quantum mechanics but only recently [4] it has been used to establish the relation of the phase of the wave function with the physical reality.

\subsection{Experimental Evidences}

Experimental confirmations of MP causality can be found in various fields of physics. Some of the most convincing results will be shortly summarized below, subject to a careful inspection, because the experiments were not particularly designed to study the MP causality. Interference of matter waves is a basic quantum mechanical phenomenon. The general outcome of mater wave experiments is that change of the MP affects the interference picture from matter waves and it can be observed experimentally. The phase sensitive experiments can be put into experiments with bound intraatomic/intramolecular wave-packets and experiments with free wave-packets.

The first case can be distinguished in an analog of Young's double slit interferometer within an atom [7], or Michelson interferometer within a molecule [8]. In these experiments, the light beams in the usual optical interferometers are replaced by electron (within atom) or nuclear (within molecule) wave packets. The wave packets are created by a sequence of usually two (pump) laser pulses of controllable phase. The wave packets evolve inside the quantum system and may overlap and interfere. A third (probe) laser pulse is used to probe the superposition of the wave packets. The interference can be probed by ion current due to ionization of atom/molecule from the superposition state [7] or by fluorescence interferogram from a higher lying excited state [8]. The local population of the interference state depends on the MP acquired by the wave packets during their evolution. The general outcome from these experiments is that change of the MP leads to observable effects: change of the fluorescence interferogram, ion current, etc., [7] [8]. In particular, even a constant phase shift of the phase-locked laser pulses (that is transferred to the MP of the wave packets) leads to observable results [7] [8].

The interference of free particles (atoms, molecules, etc.) [9]-[11] is basic phenomenon in the matter wave interferometry. Changing the phase of the atomic waves by means of 1) change of the phase of the laser fields used to split and recombine the atomic beam in the interferometer, 2) ac-Stark shift, or 3) rotation of the atomic interferometer (Sagnac effect) is well known observable result in the matter wave interferometry [10]. Free electron wave-packets interference has been recently observed experimentally employing cutting edge ultrafast physical methods [11]. The free electrons are produced ionizing argon atoms by attosecond pulses. The MP of the electron wave packets is manipulated by a momentum transfer from intense femtosecond pulses or changing the time delay between attosecond and femtosecond pulses. It leads to observable change of the interference picture [11].

\section{Dynamics-Statistical Interpretation of Quantum Mechanics}

The special theoretical arguments reveal the MP behavior of a quantum system involved in an electromagnetic interaction. The general theoretical arguments and the experimental evidences have, to our opinion, a power of proof for the MP causality. Thus, the fundamental relation of the MP with the physical reality appears to be conclusively established on that ground. That relation is not consistent with the Copenhagen interpretation as well as with non-Copenhagen interpretations proposed so far. It suggests another interpretation of quantum mechanics.

The coupled Equations (2) and (3) play crucial role to understand what is actually founded in the quantum mechanics. The wave function is a complex construct from amplitude and phase. The action/phase $S / \Phi$ is ruled by the quantum Hamilton-Jacobi Equation (2), which is the main equation in the hydrodynamic representation that introduces dynamics to the quantum system. The amplitude $R$ is ruled by the continuity Equation (3), which plays an auxiliary role, i.e., to rule the conservation of the probability density. Hence, the dynamics (associated to the phase) has leading role with respect to the statistics (associated to the amplitude) in the quantum 
mechanics. The dynamical Equation (2) includes the quantum potential $U(\boldsymbol{r}, t)=-\left(\hbar^{2} / 2 m\right)(\Delta R / R)$, which, according to Bohm's point of view, is associated with real interaction between the wave function $\Psi$, considered as "a objectively real field", and the particle [6]. This field exerts a force on the particle by means of the quantum potential and, together with the usual interaction potential $V(\boldsymbol{r}, t)$, governs its propagation. To our opinion, the quantum potential must be referred to the probabilistic properties of the amplitude $R$ rather than to a real interaction and, instead of "quantum potential", it must be more reasonably called statistical term. The statistical term associates statistical properties to the quantum dynamics through the amplitude $R$. Hence, the phase/action has a quantum-dynamical meaning, which is influenced statistically by the amplitude in the statistical term. The statistical meaning of the amplitude $R$ is influenced dynamically by the phase/action in the term including velocity $\boldsymbol{v}=m^{-1} \nabla S=-m^{-1} \hbar \nabla \Phi$ [6], Equation (3). Consequently, the dynamical and the statistical properties take place simultaneously and inseparably in the quantum mechanical description of the physical reality. Such relation between the elements of the wave function is clearly expressed within the hydrodynamic representation but it is not apparent within the Schrödinger's representation. Our understandings can be put into a dynamics-statistical interpretation of quantum mechanics. The MP causality is actually founded in the quantum mechanics but it is not recognized within its Copenhagen interpretation. Hence, the Copenhagen interpretation is incomplete because it does not reveal the relation of the phase of the wave function with the physical reality. The MP becomes a missed parameter within the standard quantum mechanics. The specified here incompleteness of quantum mechanics within the Copenhagen interpretation differs from that one based on Einstein's local realism [1].

\section{Conclusion}

Theoretical and experimental evidences for existence of a fundamental relation of the phase of the wave function and the physical reality are presented. The wave function does not have a definite physical meaning but each of its elements, amplitude and phase, are causally related with the physical reality. The phase of the wave function is primarily related to the dynamics of the quantum system, influenced statistically by the amplitude. The amplitude of the wave function is primarily related to the statistical properties of the quantum system, influenced dynamically by the phase. A new dynamics-statistical interpretation of quantum mechanics is introduced on that ground.

\section{References}

[1] Einstein, A., Podolsky, B. and Rosen, N. (1935) Physical Review, 47, 777-780. http://dx.doi.org/10.1103/PhysRev.47.777

[2] Bell, J.S. (1964) Physics, 1, 195-200.

[3] Kwiat, P.G., Eberhard, P.H., Steinberg, A.M. and Chiao, R.Y. (1994) Physical Review A, 49, 3209-3220. http://dx.doi.org/10.1103/PhysRevA.49.3209

[4] Koprinkov, I.G. (2010) Material Phase Causality or a Dynamics-Statistical Interpretation of Quantum Mechanics. In: Todorov, M.D. and Christov, C.I,, Eds., AIP Conf. Proc. 1301, Melville, NY, 573-586. http://dx.doi.org/10.1063/1.3526659

[5] Koprinkov, I.G. (2001) Journal of Physics B: Atomic, Molecular and Optical Physics, 34, 3679-3688. http://dx.doi.org/10.1088/0953-4075/34/18/306

[6] Bohm, D. (1952) Physical Review, 85, 166-179. http://dx.doi.org/10.1103/PhysRev.85.166

[7] Noel, M.W. and Stround Jr., C.R. (1995) Physical Review Letters, 75, 1252-1255. http://dx.doi.org/10.1103/PhysRevLett.75.1252

[8] Scherer, N.F., et al. (1991) Journal of Chemical Physics, 95, 1487-1511. http://dx.doi.org/10.1063/1.461064

[9] Scully, M.O., Englert, B.-G. and Walther, H. (1991) Nature, 351, 111-116. http://dx.doi.org/10.1038/351111a0

[10] Riehle, F., Witte, A., Kisters, Th. and Helmeke, J. (1992) Applied Physics B, 54, 333-340. http://dx.doi.org/10.1007/BF00325376

[11] Remetter, T., et al. (2006) Nature Physics, 2, 323-326. http://dx.doi.org/10.1038/nphys290 